Geld, Wachstum und Nachhaltigkeit

\section{Gibt es einen Wachstumszwang durch das Geldsystem?}

\author{
Unser heutiges Geldsystem scheint isoliert betrachtet keinen \\ direkten Wachstumszwang zu verursachen. Der systemische \\ Zusammenhang des Geldsystems mit anderen Treibern \\ wirtschaftlichen Wachstums sollte hingegen genauer unter- \\ sucht werden. \\ Von Markus Duscha
}

S o wie bisher kann unsere Weltwirtschaft nicht weiter wachsen, ohne dass wir uns selbst die natürlichen Grundlagen entziehen (siehe dazu u. a. Randers/Maxton 2016). Fragen nach der Qualität des Wachstums (OECD 2011) oder wie eine sogenannte Postwachstumsgesellschaft ohne wirtschaftliches Wachstum gestaltet werden könnte, werden intensiv debattiert (zum Postwachstumsdiskurs siehe zum Beispiel Seidl/ Zahrnt 2010). Als mögliche Wachstumstreiber werden unter anderem Wettbewerb, technischer Fortschritt, Wachstumspolitik, Eigentum, individuelle Motivationen oder unser heutiges Geld- und Finanzsystem diskutiert.

Die Rolle des Geldsystems für das Wirtschaftswachstum beziehungsweise eine Postwachstumsgesellschaft stand im Zentrum eines Workshops des Instituts für ökologische Wirtschaftsforschung (IÖW) sowie des gemeinnützigen Vereins für eine Geldreform, Monetative e. V., am 23. September 2016 in Berlin.

\section{Grundzüge des heutigen Geldsystems}

Im System des Euro sowie bei der überwiegenden Zahl nationaler Währungen entsteht der Großteil des Geldes durch Kreditvergabe von privaten Geschäftsbanken, das sogenannte Giralgeld. Diese Art des Geldes spielt gegenüber dem Bargeld, das von den Regierungen oder Zentralbanken geschöpft wird, heute die wesentliche Rolle: Es gab in der Eurozone Ende 2014 etwa zehnmal mehr Giralgeld als Bargeld, mit steigender Tendenz (Bundesbank 2015). Deshalb sind Kredite und das Geldsystem heute so eng miteinander verbunden.

Mit den Krediten kommen nun die Kreditzinsen ins Spiel. Diese werden als Gebühr an die Banken zusammen mit der Tilgung gezahlt [1]. Um auch den Zinsanteil der Kreditraten bedienen zu können, müssen Investoren, die einen Kredit aufnehmen, stärker wachsen, als wenn es das Kreditgeldsystem in der heutigen Form nicht geben würde (Kennedy 2012). Vor diesem Hintergrund stellt sich die Frage, ob hieraus sogar ein volkswirtschaftlicher Wachstumszwang resultiert.

\section{Forschungsansätze zu Geldsystem und Wachs- tum}

Dieser Frage widmen sich bisher wenige Ökonom/innen, denn die dominante Sicht der Ökonomik, die Neoklassik, betrachtet Geld grundsätzlich eher als neutral bezüglich des Einflusses auf die Wirtschaft.

Andere ökonomische Schulen hingegen beschäftigen sich intensiver mit dem Thema Geld und Wachstum. Richters und Siemoneit (2016) stellten verschiedene Arbeiten zusammen und untersuchten zum Beispiel mit postkeynesianischen Modellen die Frage, welche Bedingungen erfüllt sein müssten, um das Bruttoinlandsprodukt (BIP) in einem stationären Zustand zu halten [2].
Bei einer dauerhaft positiven Netto-Sparquote und positivem Zinssatz ist demnach in den Modellen ein Nullwachstum nicht möglich. Aber, so argumentieren Richters und Siemoneit, dieser Zusammenhang wäre kein geldsystemimmanenter Zwang, sondern resultiert aus Entscheidungen der Wirtschaftssubjekte: Wie viel sparen sie? Hiernach wäre ein Zwang zum Wachstum des BIP eben nicht gegeben, wenn hinreichend konsumiert würde.

Strunz et al. (2015) kommen bei ihrer Bewertung verschiedener Publikationen ebenfalls zu dem Schluss, dass ein Zusammenhang von Geldsystem und Wachstumszwang nicht zwingend wäre. Sie zeigen auf, dass vielmehr grundlegende Annahmen, die ihrer Ansicht nach zum Teil sogar den Charakter von Mythen tragen, die daraus resultierenden Ergebnisse prägen. Strunz et al. plädieren deshalb dafür, wichtige Grundannahmen kritischer aus verschiedenen ökonomischen Perspektiven zu hinterfragen. Erst dadurch würden die Untersuchungen der Vielfalt und Komplexität sozialer Phänomene gerecht, so auch unserem vermeintlichen Zwang zum wirtschaftlichen Wachstum.

\section{Fazit und offene Fragen}

Die kritische Sichtung bisher vorliegender ökonomischer Untersuchungen lässt keine gesicherte Erkenntnis zu, dass das heutige Geld-Kreditsystem einen unvermeidbaren Zwang zum Wachsen ausübt. Es bleiben aber noch viele Fragen offen, wie stark das Geldsystem insbesondere im Zusammenspiel mit anderen Aspekten als Wachstumstreiber fungiert:

I Sind geldsystemimmanente Umverteilungsaspekte von Arm zu Reich Treiber für die Wachstumspolitik? Kann also nur durch ausreichendes Wachstum vermieden werden, die durch das Geldsystem verschärften Verteilungs- und Akkumulationsfragen auf anderen Wegen wieder entschärfen zu müssen?

-Wie ist das Zusammenspiel von Geldsystem mit anderen Wachstumstrei- 
bern wie Technologieentwicklung und Wettbewerb zu beurteilen?

- Wird bei bisherigen Forschungen hinreichend berücksichtigt, dass das Geldsystem heute mehr ist als nur ein auf Krediten aufbauendes Bezahlsystem, sondern enge Verflechtungen mit der spekulierenden Finanzwelt aufweist?

- Welche Beiträge könnten in diesem Zusammenhang Geldsystem-Reformansätze spielen? Auf dem genannten Workshop am 23. September 2016 wurde diskutiert, inwiefern beispielsweise eine Vollgeldreform entschärfende Effekte auf die Wachstumsdynamik ausüben würde (Huber 2016).

- Inwieweit die dargestellten Ansätze zum Nullwachstum des BIP zugleich ökologisch und sozial vorteilhaft sind, ist eine weitere offene Frage.

Diese noch offenen Forschungsfragen sollten weiter bearbeitet werden, um die Relevanz des Geldsystems für eine nachhaltige Entwicklung besser einschätzen zu können.

\section{Anmerkungen}

[1] In diesem Beitrag wird, wie auch in den zitierten Veröffentlichungen, zunächst nur von positiven und nicht von negativen Zinssätzen ausgegangen.

[2] Betrachtete Modelle: Simple portfolio choice model by Godley/Lavoie 2012; static model by Cahen-Fourot/Lavoie (2016); stock-flow consistent input-output Model by Berg et al. 2015; stock-flow consistent model with Banks by Jackson/Victor 2015; static model revisiting Cambridge and Kalecki equations by CahenFourot/Lavoie 2016.

\section{Literatur}

Deutsche Bundesbank (Hrsg.) (2015): Geld und Geldpolitik. Frankfurt/M.

Huber, J. (2016): Monetäre Modernisierung. Vollgeld und Monetative. 5. Auflage, Marburg, Metropolis.

Kennedy, M. I. (2012): Occupy money: Creating an economy where everybody wins. Gabriola Island, B. C., New Society Publishers.

OECD (Hrsg.) (2011): Towards Green Growth. Paris, OECD-Publishing. Im Internet unter: http://dx.doi.org/10.1787/9789264111318-en (29. 9. 2016).

Randers, J./Maxton, G. (2016): Ein Prozent ist genug. Mit wenig Wachstum soziale Ungleichheit, Arbeitslosigkeit und Klimawandel bekämpfen. Bericht an den Club of Rome. München, Oekom.

Richters, O./Siemoneit, A. (2016): Consistency and Stability Analysis of Models of a Monetary Growth Imperative. VÖÖ Discussion Papers, No 1. Berlin, Vereinigung für Ökologische Ökonomie.

Seidl, I./Zahrnt, A. (2010): Postwachstumsgesellschaft. Konzepte für die Zukunft. Marburg, Metropolis.

Strunz, S./Bartkowski, B./Schindler, H. (2015): Is there a monetary growth imperative? UFZ Discussion Papers 5/2015. Leipzig, Helmholtz Zentrum für Umweltforschung UFZ.

AUTOR + KONTAKT

Markus Duscha arbeitete 25 Jahre für das ifeu Institut für Energie- und Umweltforschung Heidelberg, davon sechs Jahre als Geschäftsführer. Seit 2015 widmet er sich schwerpunktmäßig dem Themenfeld "Finanzsystem und Nachhaltigkeit".

Markus Duscha, Fair Finance Institute, Montpellierstr. 10, 69115 Heidelberg. Tel.: +496221 4263-793, E-Mail: markus.duscha@posteo.de

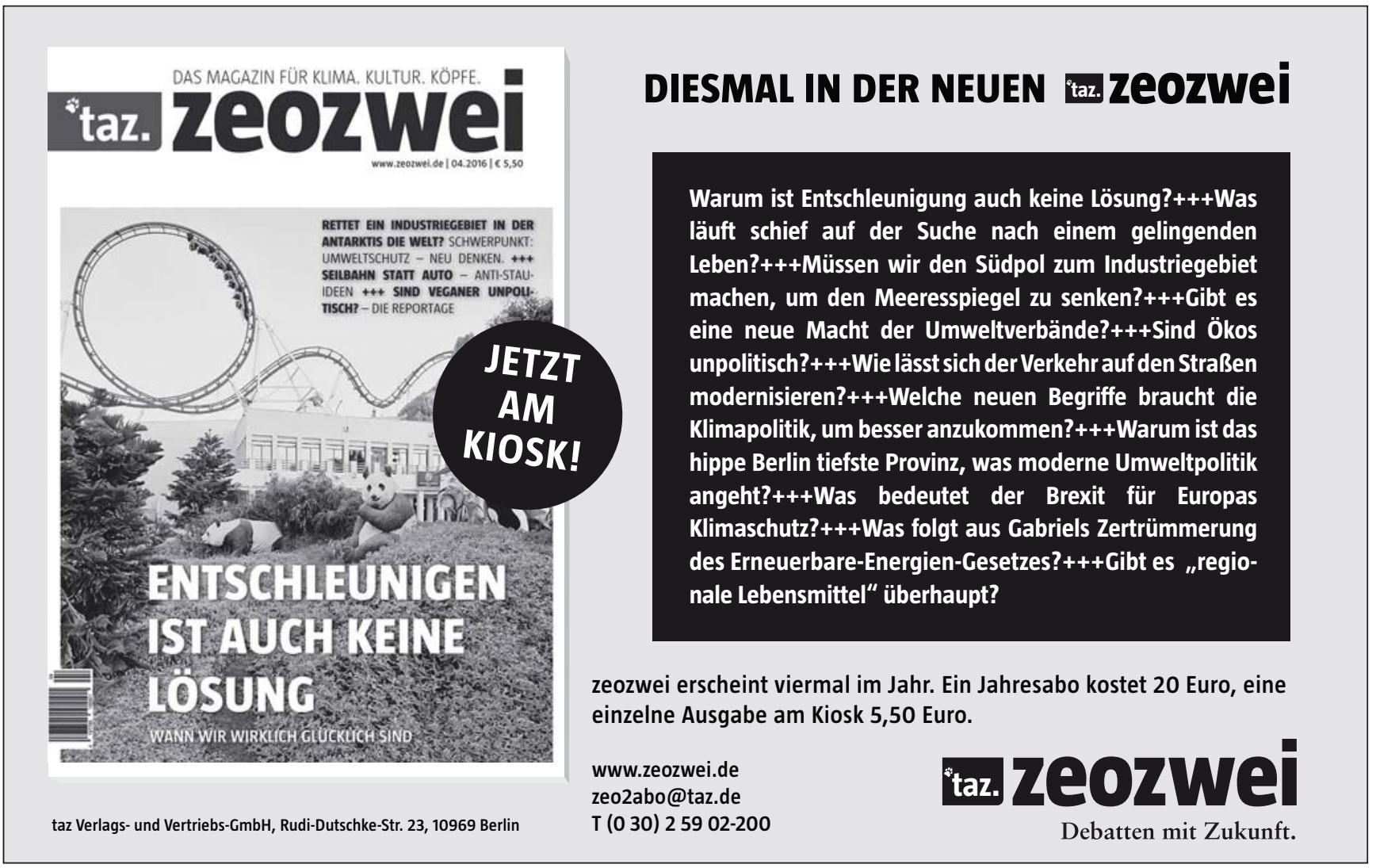

